Recepción: 20 / 04 / 2017

Aceptación: 20 / 05 / 2017

Publicación: 15 / 06 / 2017
Ciencias de la salud

Artículo de Investigación

\title{
Prótesis fija metal-porcelana en sector Anterosuperior: descripción de caso clínico
}

\author{
Fixed metal-porcelain prosthesis in the Anterosuperior sector: \\ clinical case description
}

\section{Próteses fixas no metal-porcelana maxilar anterior: Descrição de caso clínico.}

\author{
Miguel A. Rodríguez-Llaguno ${ }^{\mathrm{I}}$ \\ miguel.rodriguezll@ug.edu.ec \\ Jorge L. García-Villamar II \\ jorge.garciavi@ug.edu.ec \\ Ernesto R. Montece-Ochoa III \\ face_ermo@hotmail.com \\ Katherine E. Lima-Tamay ${ }^{\text {IV }}$ \\ kat12_1986@hotmail.com
}

Correspondencia: miguel.rodriguezll@ug.edu.ec

\footnotetext{
I. Doctor en Odontología; Diplomado en Docencia Superior; Magister en Docencia y Gerencia en Educación Superior; Universidad de Guayaquil, Guayaquil, Ecuador.

II. Doctor en Odontología; Magister en Diseño Curricular; Diplomado en Docencia Superior; Universidad de Guayaquil, Guayaquil, Ecuador.

III. Diploma Superior en Diseño Curricular por Competencias; Odontólogo; Universidad de Guayaquil, Guayaquil, Ecuador.

Iv. Doctor en Odontología; Magister en Diseño Curricular; Diplomado en Docencia Superior; Universidad de Guayaquil, Guayaquil, Ecuador.
} 


\section{Resumen}

Objetivo: Devolver la salud y equilibrio del sistema estomatognático y mejorar la estética del paciente. El sistema estomatognático nos permiten realizar acciones básicas. Sin embargo, debemos tener en cuenta que este sistema puede verse afectado, esto va a ser determinado por distintos factores que conllevan a la pérdida prematura de las piezas dentarias. Las consecuencias que podría tener un paciente parcialmente edéntulo incluyen disminución de la función masticatoria, desviación de la mandíbula, reabsorción del hueso alveolar, entre otras. El campo de la prótesis fija brinda la posibilidad de reemplazar las piezas dentarias que se han perdido a lo largo de los años. Existen estudios que indican que las prótesis fijas de metal porcelanas tienen una tasa de éxito del $85 \%$ a 10 años. Conclusiones: La elección de una prótesis fija metal-porcelana para sustituir piezas perdidas en el sector anterior superior le devuelve no solo la funcionalidad al paciente, sino también la estética. El aspecto psicológico del paciente se ve favorecido. Este tipo de restauraciones puede ser realizado en tiempo y costo relativamente bajos.

Palabras claves: Prótesis fija, piezas dentarias, paciente parcialmente edéntulo. 


\begin{abstract}
Objective: To return the health and balance of the stomatognathic system and improve the aesthetics of the patient. The stomatognathic system allows us to perform basic actions. However, we must bear in mind that this system can be affected, this will be determined by different factors that lead to premature loss of teeth. The consequences that a partially edentulous patient could have include decreased masticatory function, jaw deviation, alveolar bone resorption, among others. The field of fixed prosthesis provides the possibility of replacing teeth that have been lost over the years. There are studies indicating that metal porcelain fixed prostheses have a success rate of $85 \%$ at 10 years. Conclusions: The choice of a metal-porcelain fixed prosthesis to replace missing pieces in the anterior superior sector returns not only the functionality to the patient, but also the aesthetics. The psychological aspect of the patient is favored. This type of restorations can be performed at relatively low time and cost.
\end{abstract}

Key words: Fixed prosthesis, dental pieces, partially edentulous patient. 


\section{Resumo}

Objetivo: Para restaurar a saúde e equilíbrio do sistema estomatognático e melhorar a estética do paciente. O sistema estomatognático nos permitem executar ações básicas. No entanto, devemos considerar que este sistema pode ser afetado, isso será determinado por vários fatores que levam à perda prematura dos dentes. As consequências potenciais de um doente parcialmente desdentados incluem a diminuição da função mastigatória, desvio da reabsorção do osso alveolar da mandíbula, entre outros. O campo da prótese fixa fornece a capacidade de substituir os dentes que foram perdidos ao longo dos anos. Estudos indicam que porcelanas prótese de metal fixo tem uma taxa de sucesso de 85\% em 10 anos. Conclusões: A escolha de uma prótese fixa GFP substituir perdidos nas partes anteriores superiores não só restaura a funcionalidade para o paciente, mas também estética. O aspecto psicológico do paciente é favorecida. Este tipo de restauração pode ser feito em tempo e custo relativamente baixo.

Palavras-chave: prótese fixa, dentes, paciente parcialmente desdentado. 


\section{Introducción.}

El sistema estomatognático es un complejo conjunto de órganos que nos permiten realizar acciones básicas como comunicarse, alimentarse, etc. Sin embargo, este sistema puede verse afectado por distintas causas, como lo son: caries, enfermedad periodontal, etc., que provocan la pérdida prematura de las piezas dentarias. (Colque, 2005)

A pesar de los avances en el campo de la Odontología para preservar la salud bucal de los pacientes, no se ha podido evitar la pérdida de piezas dentarias. Las consecuencias que podría tener un paciente parcialmente edéntulo incluyen disminución de la función masticatoria, desviación de la mandíbula, reabsorción del hueso alveolar, alteraciones estéticas, con lo cual también puede verse afectada la psicología de esta persona. (Sánchez, Troconis, \& Di Geronimo, 1998)

Es así como surge la necesidad de implementar procedimientos clínicos encaminados a solucionar esta problemática. El campo de la prótesis fija brinda la posibilidad de reemplazar las piezas dentarias que se han perdido a lo largo de los años. (Sánchez, Troconis, \& Di Geronimo, 1998)

Si se han perdido varias piezas dentales, una buena opción es un puente fijo permanente. Para realizar una prótesis de este tipo se requiere de un proceso largo. (Pico Cid, Nieves Cervantes, Ramón Jiménez, \& González Heredia, 2009)

En 2006, Corts dijo: "las prótesis fijas metal-cerámica o metal-resina han sido por décadas procedimientos confiables y de amplia utilización por la profesión. (Corts, 2006)

El éxito de un puente fijo permanente se debe no solo al conocimiento y habilidad del rehabilitador. Es importante realizar un diagnóstico y plan de tratamiento personalizado en cada paciente. Según Watanabe y colegas (2008), "la planificación presurosa, segmentada, que ignora los 
principales aspectos del tratamiento requerido, desafía los modernos conceptos de tratar al paciente en su totalidad, más que a los dientes individuales”. (Watanabe, y otros, 2008)

Cada uno de los pasos clínicos tales como la preparación de los dientes pilares, impresiones, pruebas y cementado deben realizarse siguiendo los principios básicos en este tipo de rehabilitación. De lo contrario, la probabilidad de fracaso es mayor. (Vargas \& Ornes, 2013)

Existen estudios que indican que las prótesis fijas de metal porcelanas tienen una tasa de éxito del $85 \%$ a 10 años. Esto nos permite evaluar la viabilidad de realizar un procedimiento de este tipo. (Vilarrubí, Pebé, \& Rodríguez, 2011).

\section{Materiales y métodos.}

\section{Presentación del caso clínico}

Paciente de sexo femenino, 47 años de edad, asiste a a la facultad Piloto de odontología de la universidad de Guayaquil, su motivo de consulta era la falta de piezas dentarias en el sector anterior y la queja por la apariencia estética de su sonrisa.

Al examen clínico se observan buenas condiciones de salud bucal. Presenta zonas edéntulas parciales que involucra las piezas $11,21,12,22,13,23,14,24$, que consiste en una brecha de mediana extensión. Las pizas 13, 23, 14, 24 que se tomaron como pilares presentan buena posición y orientación en relación a los arcos dentarios y salud periodontal. Las coronas de las piezas pilares presentan buen volumen y atura gingivo-oclusal: la relación oclusal con los antagonistas naturales es correcta.

\section{Diagnóstico}

Ausencia de piezas dentarias: 11, 21, 12, 22, 13, 23, 14, 24 en el sector anterior superior. 
Miguel A. Rodríguez-Llaguno; Jorge L. García-Villamar; Ernesto R. Montece-Ochoa; Katherine E. Lima-Tamay

Plan de tratamiento

Elaboración de una prótesis parcial fija metal-porcelana.

\section{Resultados.}

Procedimiento preclínico de la confección de los provisionales de acrílico.

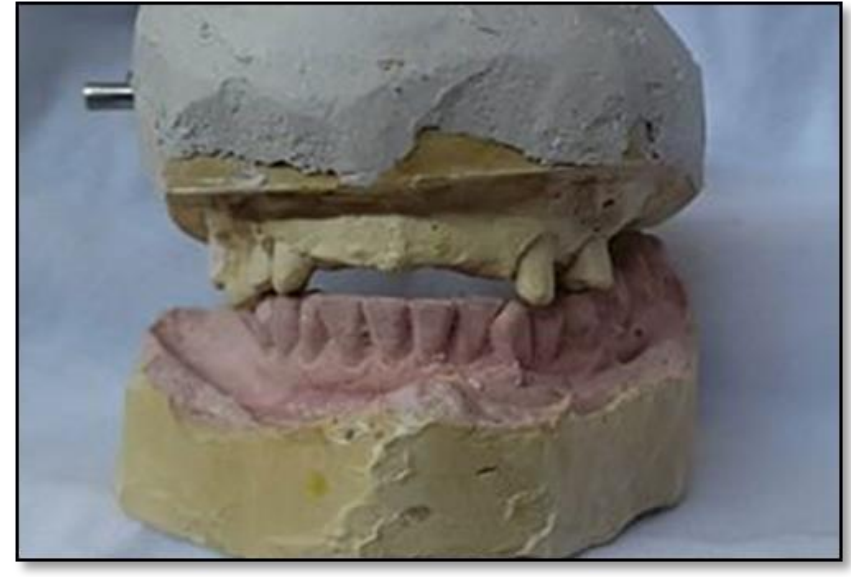

Modelo de estudio montado en oclusador.

\section{Imagen 1}

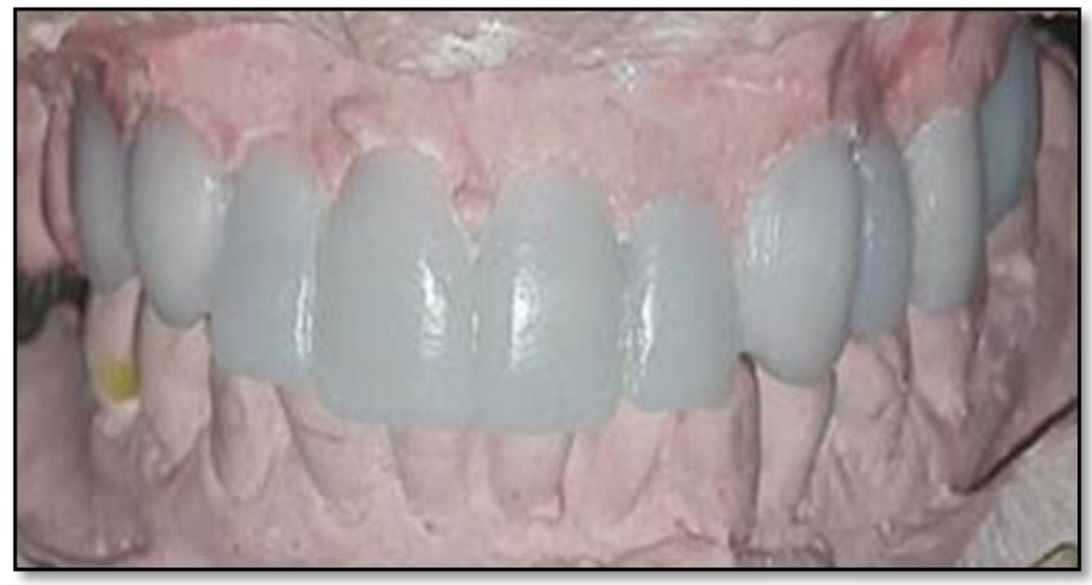

Modelo de estudio con el encerado de diagnóstico de los dientes faltantes.

Imagen 2 


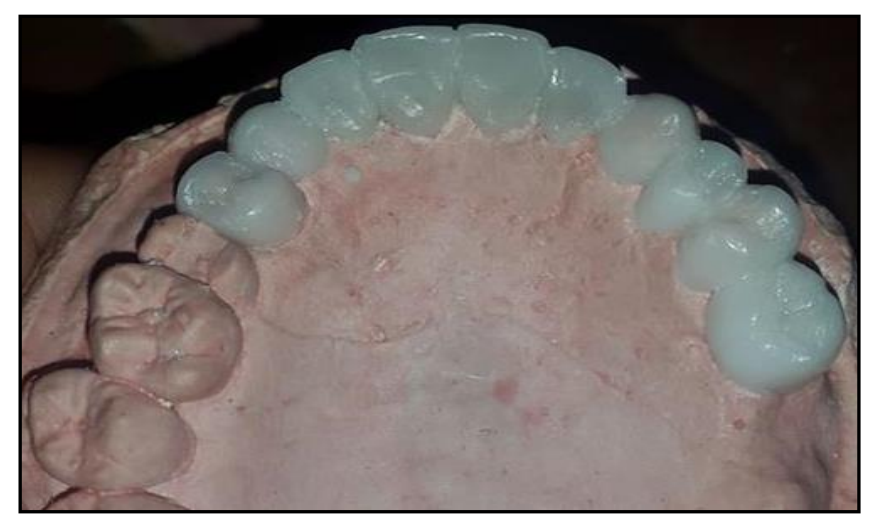

Modelo de estudio con el encerado de diagnóstico de los dientes faltantes.

Imagen 3

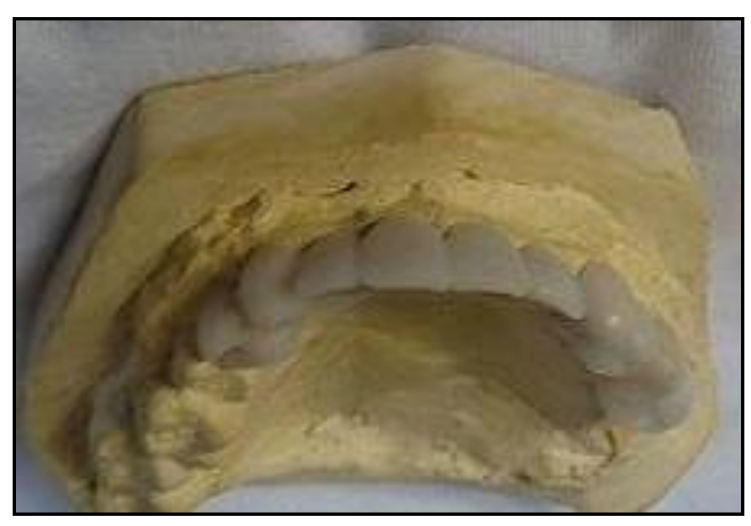

Modelo de estudio con el encerado de diagnóstico de los dientes faltantes.

Imagen 4

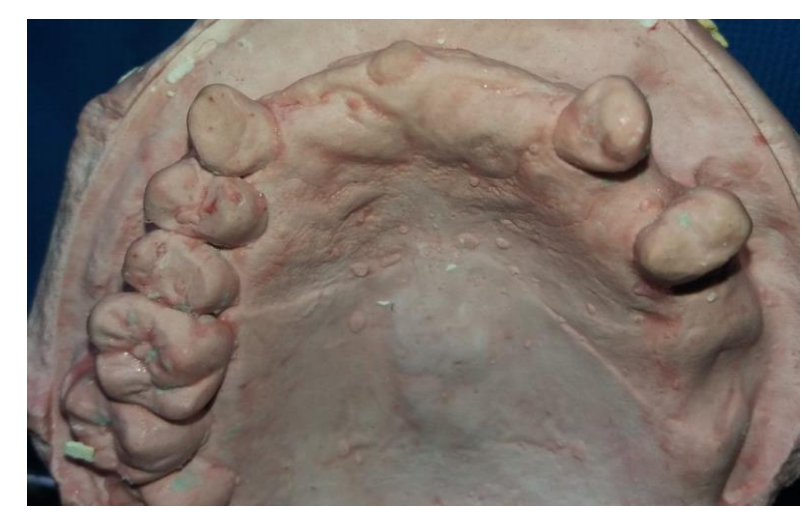

Modelo de estudio con la preparación de coronas completas de los dientes que van a servir como pilares de un puente.

Imagen 5 
Miguel A. Rodríguez-Llaguno; Jorge L. García-Villamar; Ernesto R. Montece-Ochoa; Katherine E. Lima-Tamay

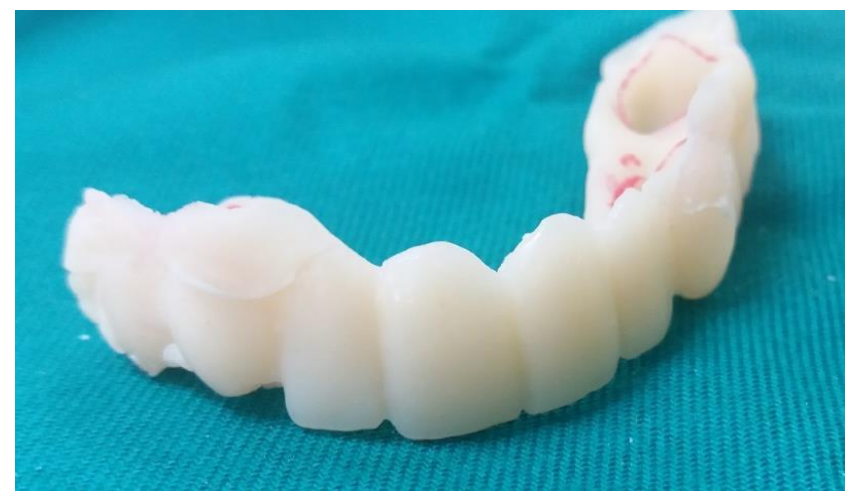

Modelo de estudio con los provisionales de acrílico. Imagen 6

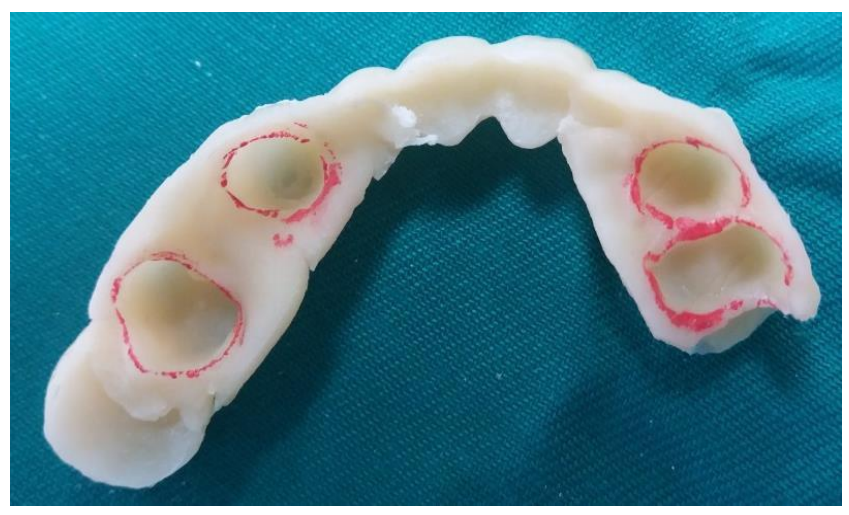

Modelo de estudio con los provisionales de acrílico.

Imagen 7

PRIMERA CITA

Procedimiento clínico del caso en paciente

Técnica de preparación de corona completa anterosuperior

1. Surco de orientación 


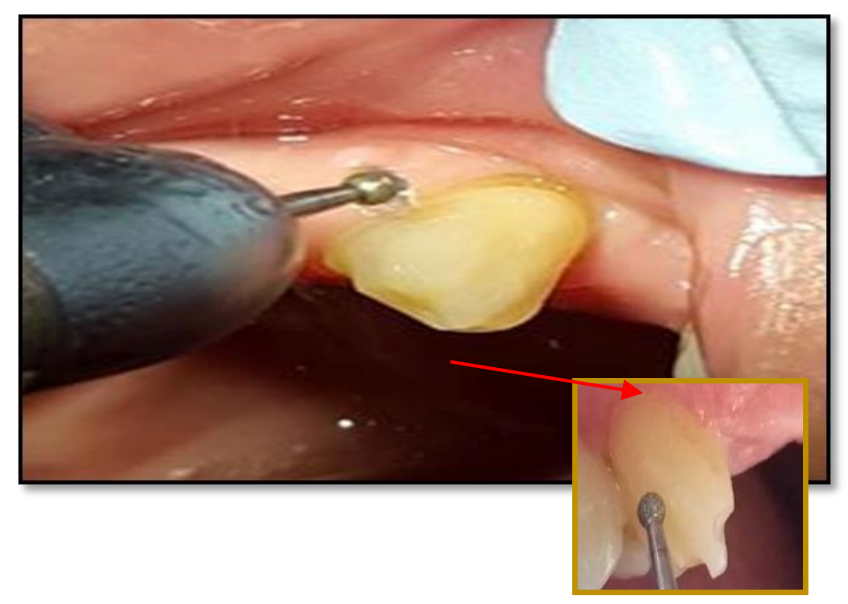

Realización de los surcos de orientación (mesial-medio-distal) en la cara vestibular: con una fresa de diamante redonda mediana.

\section{Imagen 8}

2. Reducción de la cara vestibular

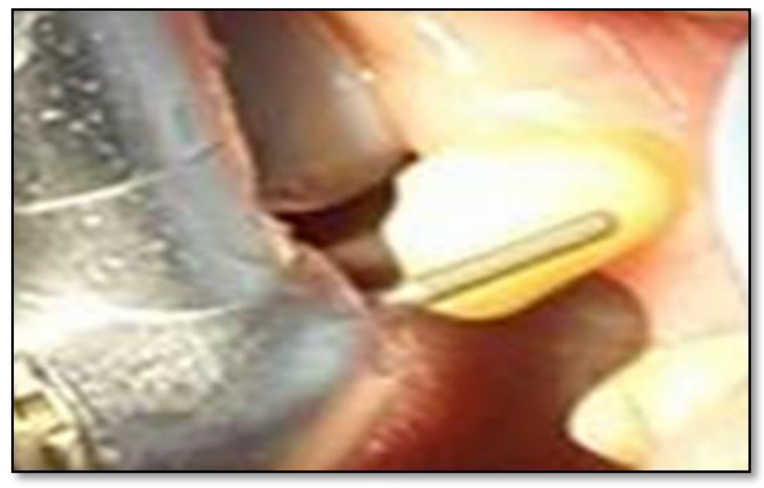

Reducción de la cara vestibular con una fresa de diamante troncocónica punta redondeada. Imagen 9

3. Reducción de la cara palatina

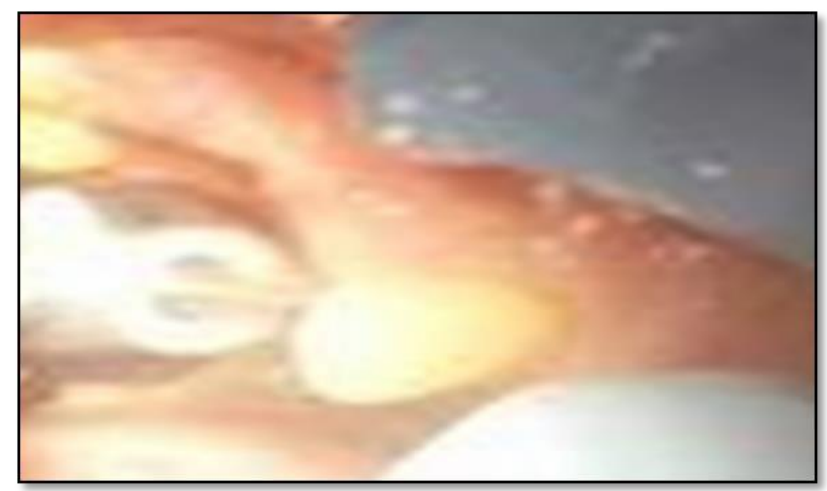

Reducción de la cara palatina con fresa de diamante de balón mediana. Imagen 10 
4. Reducción del borde incisal

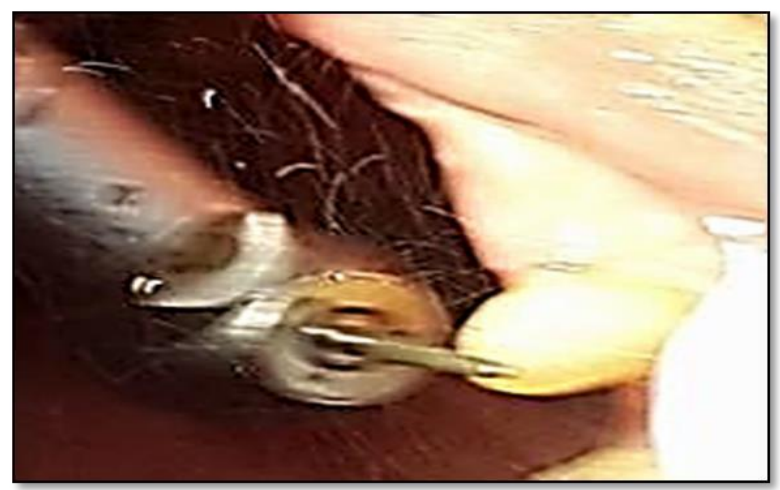

Reducción del borde incisal con fresa de diamante troncocónica punta de lápiz. Imagen 11

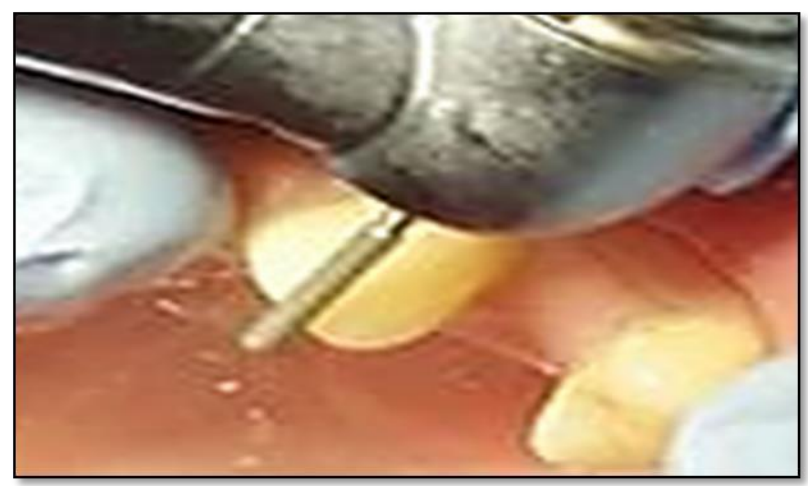

Reducción del borde incisal con fresa troncocónica punta redondeada. Imagen 12

5. Corte proximal

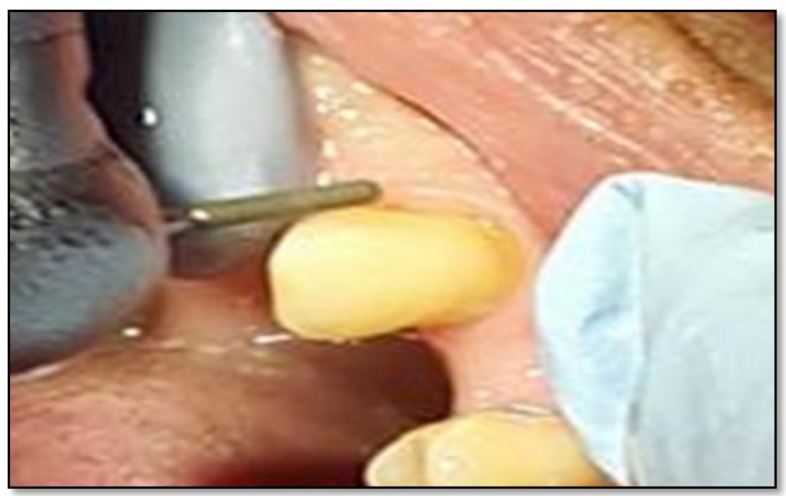

Corte proximal por cara mesial y distal con fresa de diamante troncocónica punta redondeada Imagen 13 
6. Reducción cervical

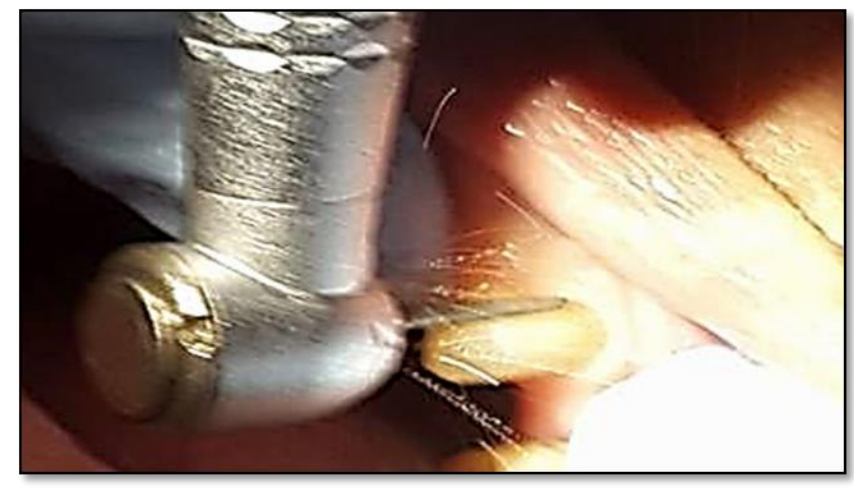

Reducción del tercio cervical con fresa diamante punta de lápiz. Imagen 14

7. Pilares dentarios

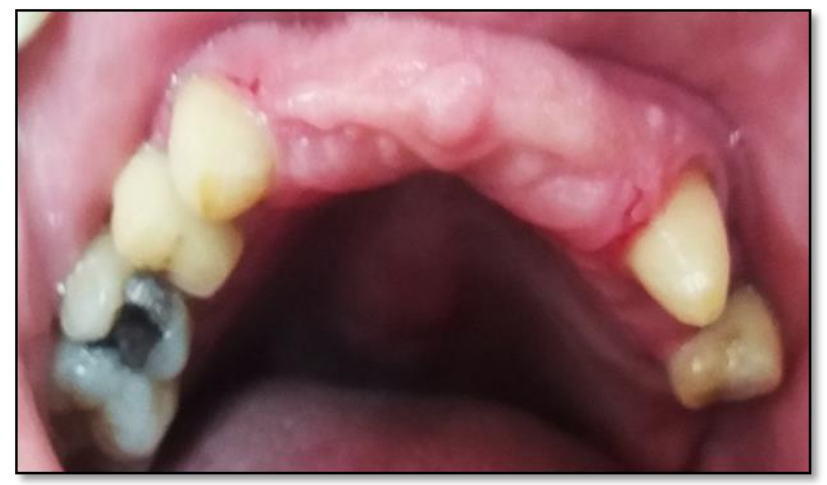

Preparación de los dientes que van a servir como pilares de un puente fijo. Imagen 15

8. Provisional de acrílico en boca del paciente

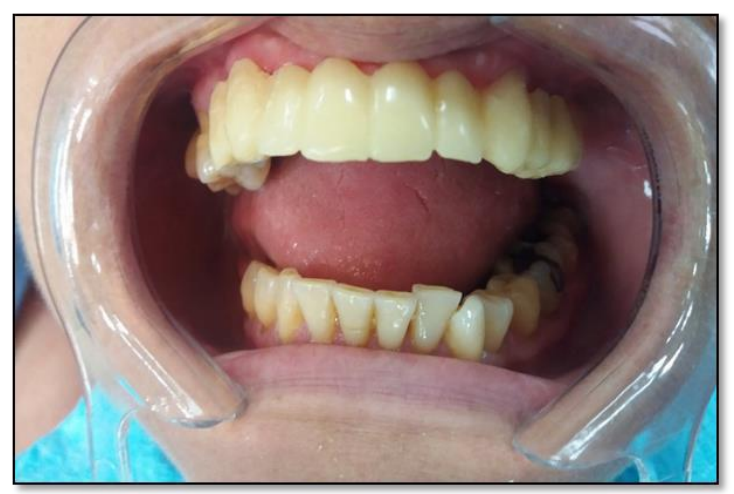

Colocación de provisional de acrílico con cemento provisional en piezas pilares y ausentes. Imagen 16 
Miguel A. Rodríguez-Llaguno; Jorge L. García-Villamar; Ernesto R. Montece-Ochoa; Katherine E. Lima-Tamay

9. Retiro de excesos de cemento provisional

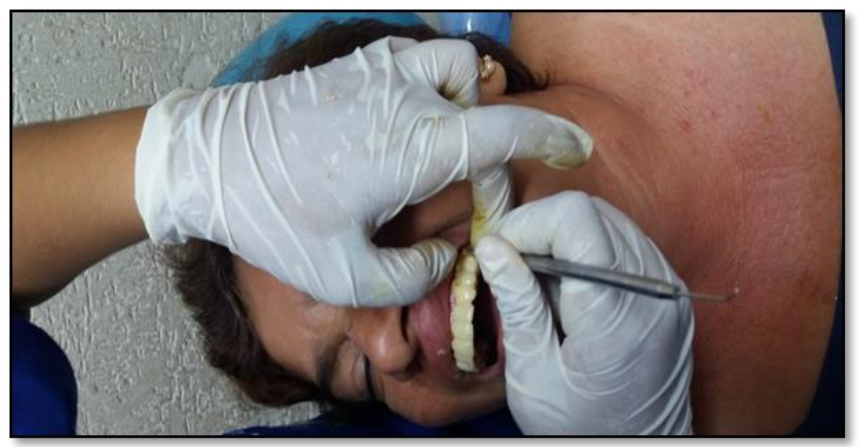

Retiro de excesos de cemento provisional con cucharilla.

Imagen 17

SEGUNDA CITA

10. Colocación de hilo retractor en dientes pilares.

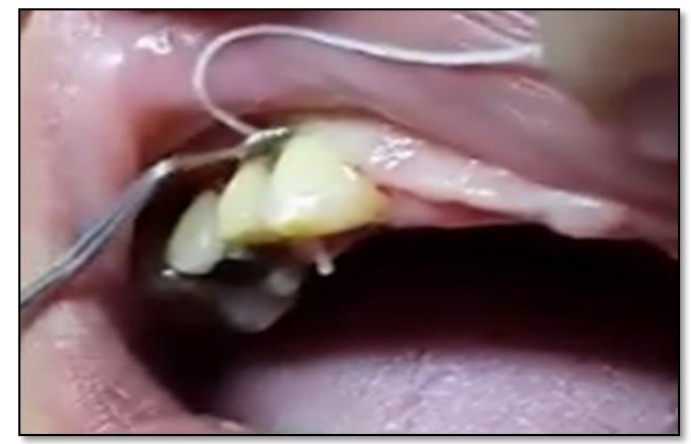

Hilo retractor en pieza pilar 15.

Imagen 18

11. Impresión con silicona de adición

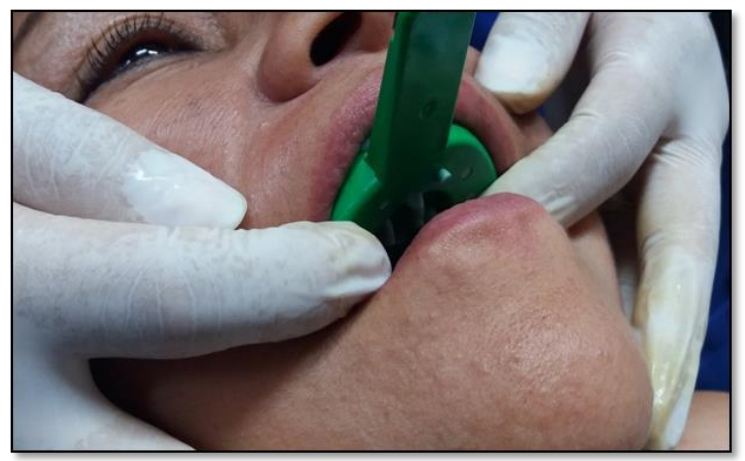

Impresión con material pesado.

Imagen 19 


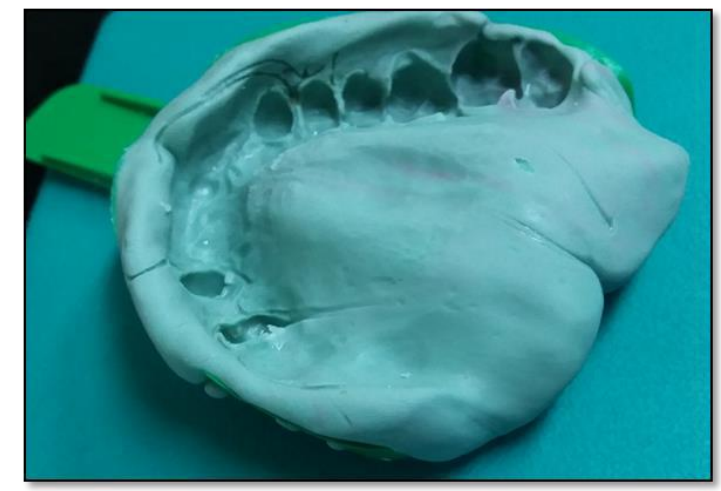

Impresión con material pesado de adición. Imagen 20

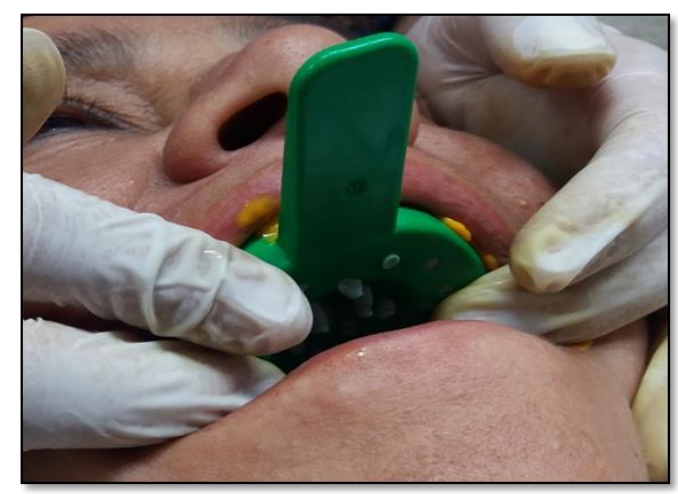

Toma de impresión con Silicona de adición: material liviano. Imagen 21

12. Troquelado en modelo de estudio y metal.

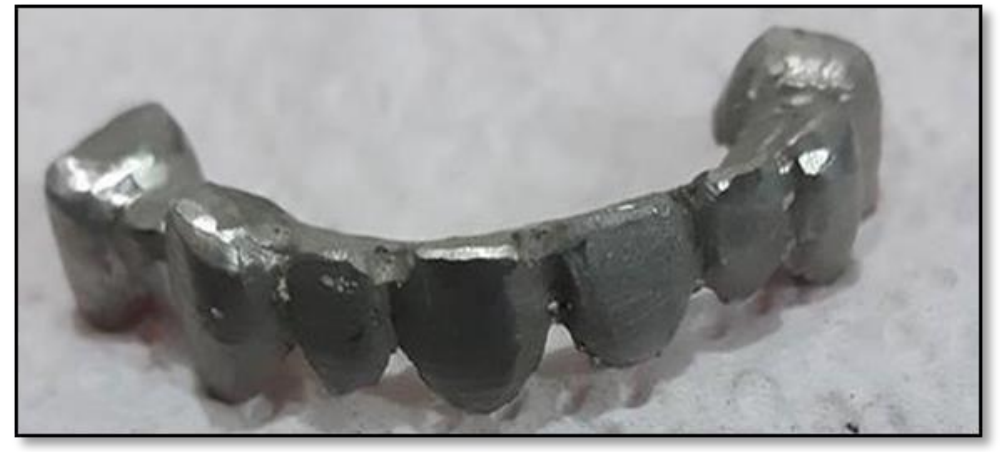

Imagen 22 
Miguel A. Rodríguez-Llaguno; Jorge L. García-Villamar; Ernesto R. Montece-Ochoa; Katherine E. Lima-Tamay

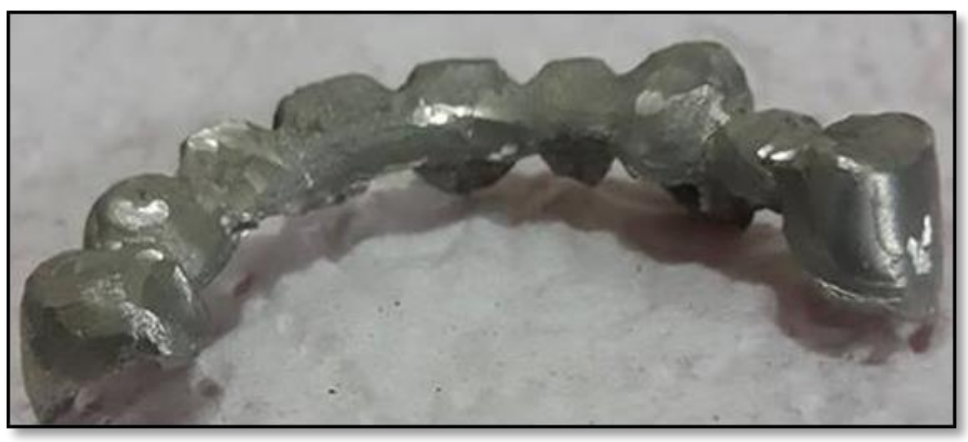

Imagen 23

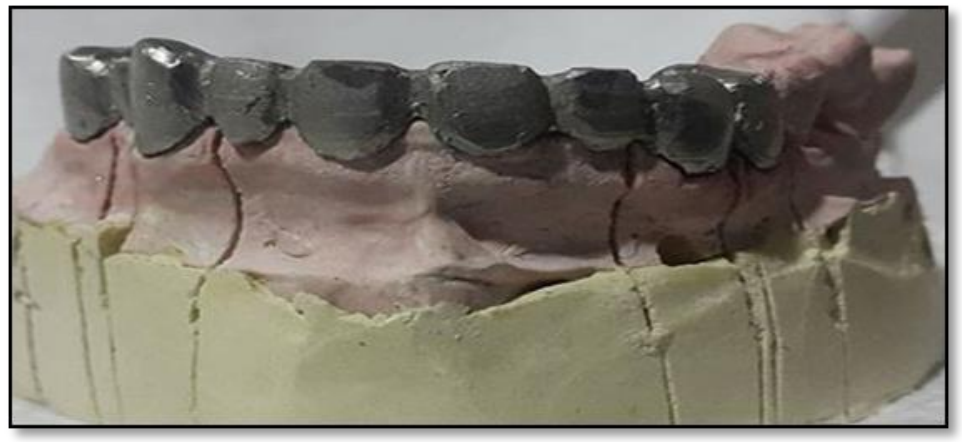

Imagen 24

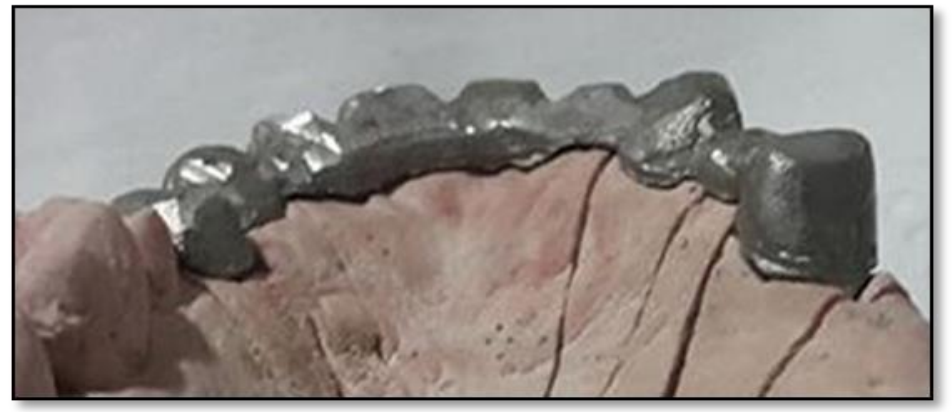

Troquelado y metal en modelo de estudio.

Imagen 25

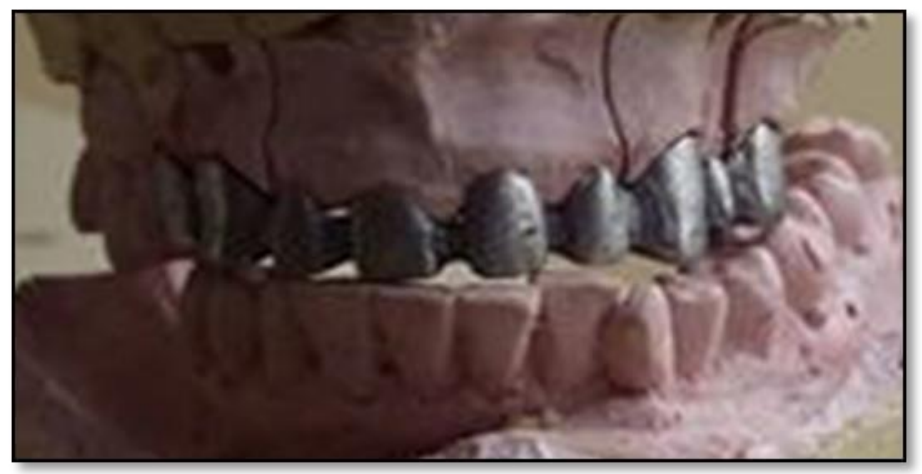

Imagen 26 


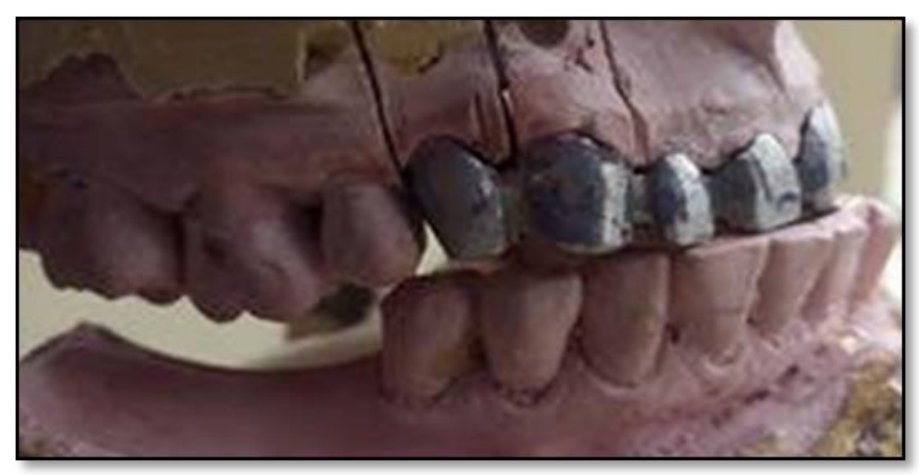

\section{Imagen 27}

13. Prueba del metal y adaptación en boca del paciente.

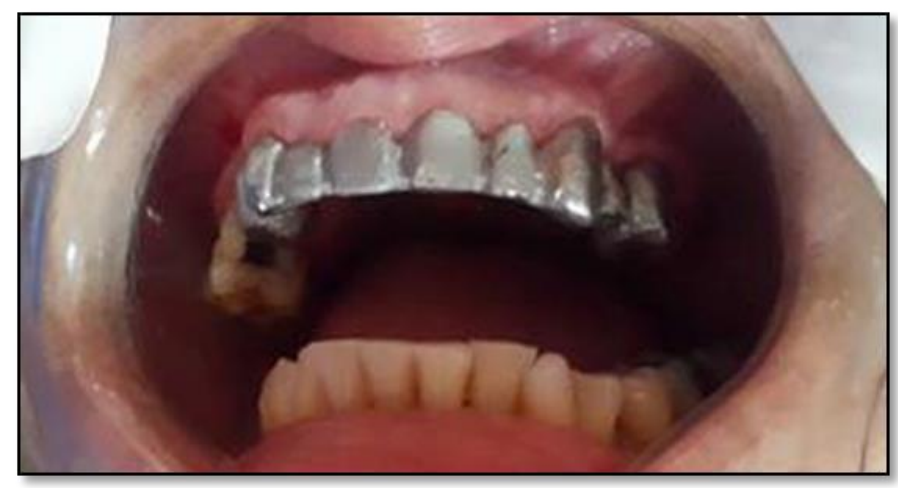

\section{Imagen 28}

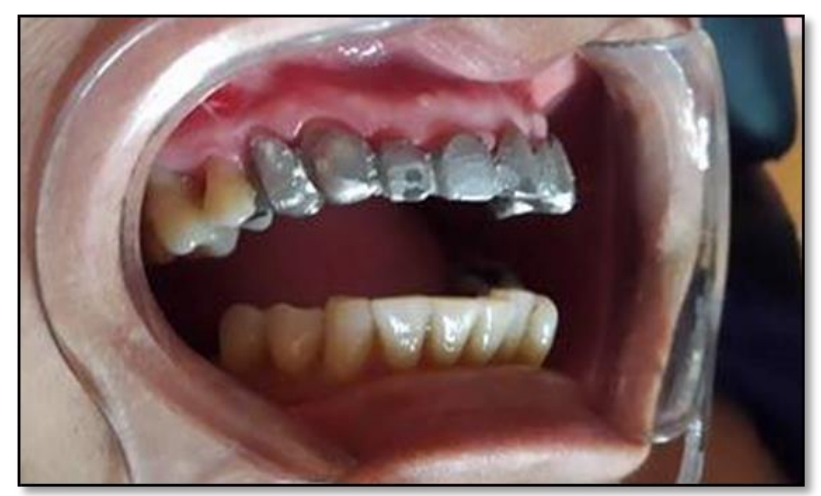

Imagen 29 
Miguel A. Rodríguez-Llaguno; Jorge L. García-Villamar; Ernesto R. Montece-Ochoa; Katherine E. Lima-Tamay

\section{Tercera cita}

14. Prueba del biscocho de porcelana y adaptación en boca del paciente.

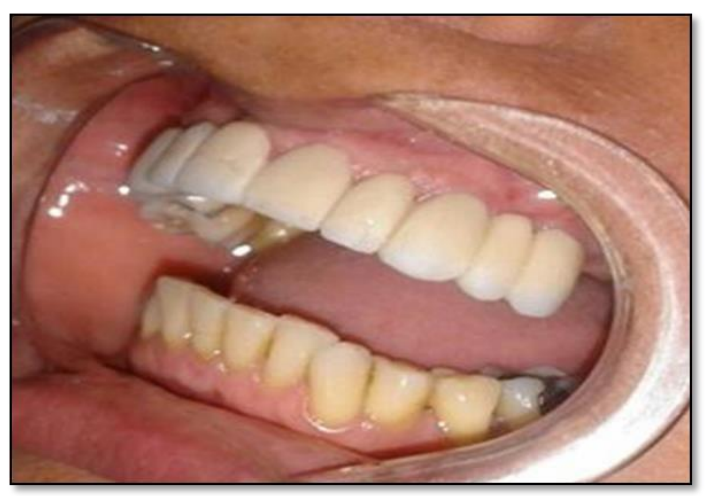

\section{Imagen 30}

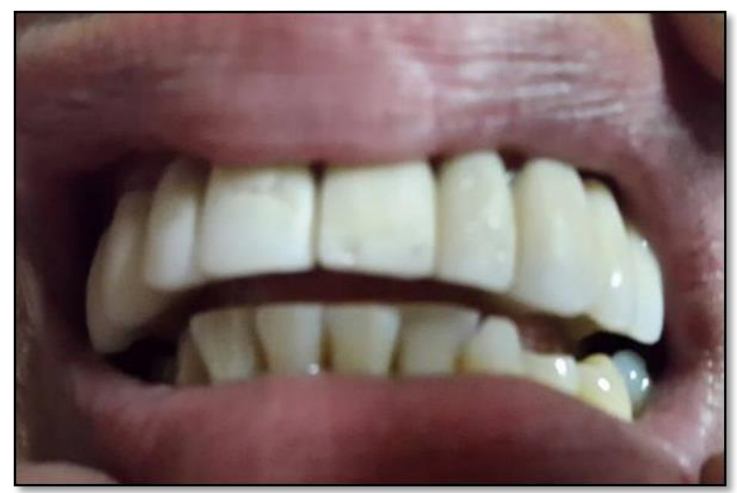

\section{Imagen 31}

15. Adaptación y cementación de puente fijo metal-porcelana.

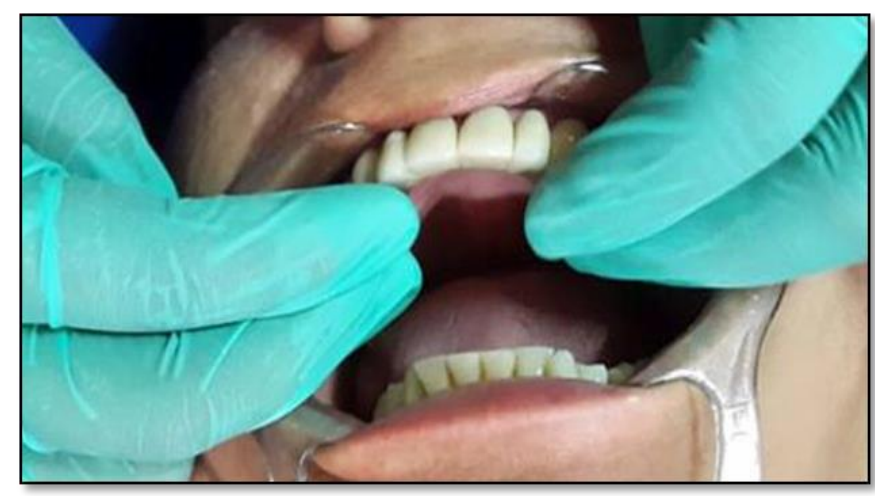

Imagen 32 


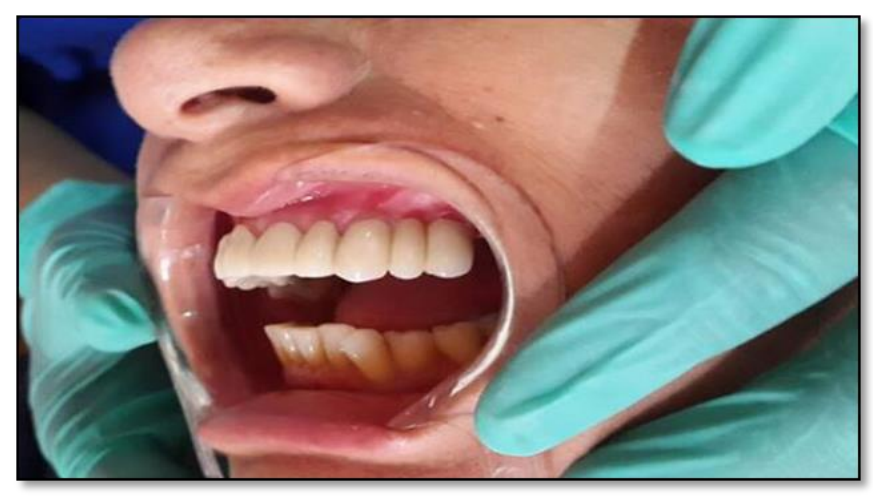

\section{Imagen 33}

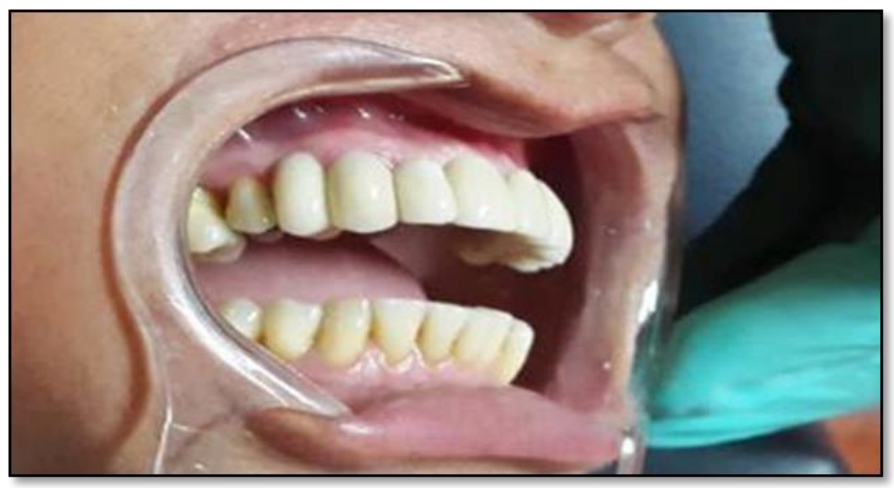

Imagen 34

16. Paciente rehabilitada con puente fijo metal porcelana.

\section{Conclusiones.}

La restauración de las piezas perdidas mediante una prótesis fija metal-porcelana en el sector anterior superior devuelve al paciente no solo la funcionalidad. Esta zona exige un alto grado de estética. El puente de metal porcelana brinda las características necesarias para que el paciente pueda regresar a un estado de salud de su cavidad bucal.

Esto nos demostró que a pesar de que las preparaciones en los dientes que servirán como pilares incluyan la eliminación de tejido sano, el resultado final tiene un impacto positivo en el paciente, incluso en su estado psicológico. 
Este tipo de restauraciones se las realiza en un periodo de tiempo relativamente corto y a un valor económico bajo, si se lo compara con procedimientos más invasivos como la colocación de implantes intra óseos.

\section{Bibliografía.}

Colque, A. (junio de 2005). Obtenido de http://www.oocities.org/boliviadental/artic/terminaciones.html

Corts, J. (2006). Prótesis fija de resina compuesta reforzada con fibra/cerómero con carilla cerámica asociada. Revista de Operatoria Dental y Biomateriales.

Pico Cid, A., Nieves Cervantes, C., Ramón Jiménez, R., \& González Heredia, E. (2009). Puente fijo de resina autopolimerizable a un año de uso sin fallas. MEDISAN.

Sánchez, A., Troconis, I., \& Di Geronimo, M. (1998). Necesidades protésicas de los pacientes que asisten a la Facultad de Odontología de la Universidad Central de Venezuela. Acta Odontológica Venezolana.

Vargas, Y., \& Ornes, M. (2013). Caso clínico de rehabilitación protésica fija: puente zirconio sector anterior superior. iDental.

Vilarrubí, A., Pebé, P., \& Rodríguez, A. (2011). Prótesis convencional libre de metal: Tecnología CAD CAM-Zirconia, descripción de un caso clínico. Odontoestomatología.

Watanabe, R., Salcedo, D., Ochoa, J., Horna, H., Herrera, M., \& Paz, J. (2008). Rehabilitación oral con prótesis fija. Odontología Sanmarquina, 96-99.

Hospichannel Cancun; Hospichannel Cancun; 28 noviembre 2014; Protesis Dental Fija; URL: https://www.youtube.com/watch?v=f8cwyjCBsQg

Wilber Guillermo Abregu Canales; wilber guillermo abregu canales; 31 octubre 2012; puente estético de porcelana, mundo dental sur; URL: https://www.youtube.com/watch?v=iSgmSLCFLOU

Sara Maria Jimenez Estrada; Sara Maria Jimenez Estrada; 30 octubre 2013; Rehabilitación con prótesis fija, removible y aditamentos de semiprecision; URL: https://www.youtube.com/watch?v=1q97a1wR8Lg 